\title{
Relationship between vestibulo-ocular reflex gain and dizziness handicap inventory score to predict effectiveness of vestibular rehabilitation
}

\author{
Morteza Hamidi Nahrani ${ }^{\circledR}$, Mehdi Akbari ${ }^{*}{ }^{(\mathbb{D}}$, Mohammad Maarefvand \\ Department of Audiology, School of Rehabilitation Sciences, Iran University of Medical Sciences, Tehran, Iran
}

Received: 10 Apr 2021, Revised: 9 May 2021, Accepted: 14 Jun 2021, Published: 15 Oct 2021

\begin{abstract}
Background and Aim: Evaluating the effectiveness of vestibular rehabilitation (VR) in patients with vestibular lesions has always been a challenge. The questionnaires that are used for this purpose mostly show the degree of vestibular disability rather than providing information about improvement of vestibular dysfunction. This study aimed to evaluate whether video head impulse test (vHIT) that is used for the examination of vestibulo-ocular reflex (VOR), is a useful method for predicting the effectiveness of VR and has a correlation with dizziness handicap inventory (DHI) score.
\end{abstract}

Methods: Participants were 42 patients with unilateral peripheral vestibular hypofunction (UPVH) undergoing VR. Patients were assessed before and after rehabilitation by the vHIT in all ipsilesional and contralesional semicircular canals (SCCs) and the DHI. The changes in DHI score and VOR gain before and after rehabilitation, were shown as $\Delta \mathrm{DHI}$ and $\Delta \mathrm{VOR}$ and their correlation was evaluated.

Results: VOR gain from ipsilesional and contralesional SCCs was improved significantly after

\footnotetext{
* Corresponding author: Department of Audiology,

School of Rehabilitation Sciences, Iran University of

Medical Sciences, Shahid Shahnazari St., Madar

Square, Mirdamad Blvd., Tehran, 15459-13487, Iran.

Tel: 009821-22228051,

E-mail: Akbari.me@iums.ac.ir
}

VR. There was a significant strong negative correlation between $\triangle \mathrm{VOR}$ gain from ipsilesional SCCs and $\triangle \mathrm{DHI}$ score but no significant correlation was found between the $\triangle \mathrm{DHI}$ score and $\triangle$ VOR gain from contralesional SCCs.

Conclusion: vHIT test is a useful tool to evaluate the effectiveness of VR. VOR gain is correlated with the DHI score. Therefore, the improvement in VHIT results in all three SCCs after VR may be a good predictor of the degree of improvement in dizziness-related disability.

Keywords: Vestibular rehabilitation; follow-up; unilateral vestibular hypofunction; video head impulse test; dizziness handicap inventory

\section{Citation: Hamidi Nahrani M, Akbari M,} Maarefvand M. Relationship between vestibuloocular reflex gain and dizziness handicap inventory score to predict effectiveness of vestibular rehabilitation. Aud Vestib Res. 2021;30(4):273-9.

\section{Introduction}

Recently, there has been a growing interest in the use of vestibular rehabilitation (VR) for the management of unilateral peripheral vestibular hypofunction (UPVH) which refers to a unilateral damage to peripheral vestibular structures including semicircular canals (SCCs), resulting in vestibulo-ocular reflex (VOR) asymmetry. Static 
symptoms such as spontaneous nystagmus and postural abnormalities are eliminated within a few weeks; however, patients continue to have difficulty with dynamic visual activities during head movements due to inadequate central compensatory mechanisms. This condition is associated with vertigo, balance problems, loss of visual stability, and blurred vision, which significantly affect the daily living activities [1-5]. VR is an exercise-based therapy that improves active dynamic visual acuity during head movements due to VOR gain [6,7]. Evaluating the effectiveness of VR has always been challenging $[8,9]$. Questionnaires survey patients' general conditions and degree of disability, and are not designed to examine vestibular improvement. On the other hand, most balance tests are not suitable for the examination of peripheral vestibular dysfunction and or cannot monitor the rehabilitation process $[10,11]$. There are several studies that have shown the effect of treatment in patients with vestibular dysfunction. Studies have confirmed the acceptability of the dizziness handicap inventory (DHI) questionnaire in monitoring the rehabilitation process $[12,13]$. Despite many strengths, DHI has some limitations. It is influenced by factors not related to vestibular lesions, such as age, socioeconomic status, cognitive ability, physical activity, and metabolic diseases such as diabetes. Therefore, this questionnaire may not reflect the extent of vestibular damage or improvement in vestibular function after rehabilitation [10]. Moreover, the DHI scores are influenced by patients' subjective responses and documented disabilities, may be due to other underlying conditions [14]. Evaluating the VR results using an objective vestibular test may reduce the DHI limitations. Objective assessment of vertigo has always been a clinical challenge. Objective vestibular tests show the extent of the lesion in the affected area and are more accurate than the questionnaires that only indicate the patients' overall disability [15]. Previous studies have used posturography and caloric test to predict disability caused by vestibular lesions. The caloric test is not suitable for monitoring the rehabilitation process because it is unable to distinguish between static and dynamic recovery [16]. The posturography test shows the general balance ability of the subjects and is not suitable for the examination of vestibular lesions, especially peripheral ones [17]. Therefore, none of these tests can be used specifically for monitoring the rehabilitation process of peripheral vestibular lesions. Video head impulse test (vHIT) is a specific and valid test for the evaluation of all three types of SCC. This test was first introduced by Halmagyi and Curthoys. The vHIT results obtained from VOR gain in SCCs can help with the diagnosis of visual stability disorders [18]. Two previous studies used vHIT for the follow-up of VR $[7,15]$. However, only horizontal SCCs were studied and the correlation of the questionnaire score and vHIT results was not reported after VR. The vHIT examines eye movements during highvelocity head movements, which is similar to the speed of head movements during daily living activities. Therefore, it can provide useful information about the extent of physiological dysfunction in the vestibular organ and its impact on daily life. From this perspective, vHIT can be used for the prognosis of dizziness-related disability [18]. However, some studies believe that vHIT cannot predict the degree of dizzinessrelated disability because it is also influenced by non-vestibular factors such as age and cognitive factors $[10,16]$. Hence, DHI and vHIT show two different aspects of vestibular lesions. Monitoring of study reports from different VR models is necessary to validate or modify these models. However, this process is solely based on the caloric testing, posturography, use of questionnaires, and qualitative indicators, and there is a need for quantitative evaluation of the rehabilitation outcome. In this regard, this study aimed to evaluate the effectiveness of VR using the vHIT, and to analyze the correlation between vHIT and DHI results for VR reports. Since vHIT can separately and quantitatively predict the average VOR gain in all three ipsilesional and contralesional SCCs, this study attempted to provide a better understanding of the role of vHIT in monitoring the VR process. To our knowledge, this is the first comprehensive study on the role of vHIT in VR follow-up and determination of its correlation with DHI results. 


\section{Methods}

\section{Participants}

This is a clinical trial approved by the Ethics Committee of Iran University of Medical Sciences (Code: IR.IUMS.REC.1399.727). Forty-two patients with UPVH (23 women and 19 men) with a mean age of $42.90 \pm 10.14$ years (ranged from 25-59 years) participated in the study suffering from vertigo (2-4 weeks after onset), balance problems, loss of visual stability and blurred vision who were selected randomly from outpatients referred to an audiovestibular clinic. All participants signed a written consent form and declared they were aware of the procedures and were free to participate in the study. The diagnostic criteria for UPVH were sudden onset of severe vertigo in the absence of neurological or audiological symptoms and unilateral canal paresis $>25 \%$ on bithermal caloric testing. Patients with endolymph hydrops (characterized by episodic vertigo and fluctuating hearing loss), diabetes, severe neck problems, severe visual impairment, benign positional vertigo, and central vestibular lesions (according to videonystagmography test results) [10] were excluded from the study.

\section{Intervention}

A supervised, home-based VR program was provided to the patients. The VR protocol was based on the program proposed by Herdman et al. [19]. It consisted of adaptation and eye-head exercises designed to improve gaze stability as well as gait and balance exercises. All patients received oral and written instructions to perform exercises at home three times a day at least for 30 minutes and were advised to increase the number of repetitions by $5-10$ as tolerated. The instructions were given in detail to the patients and their companions in a face-to-face session before the start of the program. The researcher contacted the patients by telephone every days and asked them about the exercise process and answered their questions. All patients were visited in the clinic weekly to review their exercise program. All communications were done for encouraging and providing consultation.

\section{Measures}

First, the Persian version of the DHI (DHI-P) was completed by the patients under the supervision of an audiologist to assess their balance problems. The DHI-P is a valid tool with a Cronbach's coefficient of 0.86 [20]. It includes 25 items answered by yes ( 4 points), sometimes ( 2 points), or no ( 0 point). The total score ranges from 0 to 100, where higher scores indicate more severe disability [21]. The patients were then assessed by the vHIT test using the Synapsys vHIT system. Subjects were asked to sit at a distance of 90 $\mathrm{cm}$ from a tripod camera and stare at a target on a wall at $2 \mathrm{~m}$ distance. The examiner stood behind the patient and rotated the patient's head unpredictably $10-20$ degrees to the right or left in the horizontal direction with the eyes fixed on the target to stimulate bilateral horizontal SCC. The peak head velocity of the impulses was gradually increased from 50 to $250 \%$ s (ranged from 5 to 20 degrees) [22]. To assess bilateral vertical right superior-left posterior (RALP), and left superior-right posterior (LARP) SCCs, the head first turned 30-45 degrees to the right and left relative to the trunk and then rotated downward (stimulating superior SCC) or upward (stimulating posterior SCC) during the impulses. The device's infrared camera estimated the velocity of eye movements relative to head velocity [22]. VOR gain from the three ipsilesional and contralateral canals was quantified between 0 and 1 . Since the lesion was unilateral and we evaluated both SCCs sides, the ipsilesional side shows the affected side and the contralesional side shows the non-affected side. VOR gain of 1 indicates the equality of head and eye movement velocities. This coordination decreases as VOR gain reduces towards 0 . Subjects with VOR gain $>1.2$ were excluded from the study due to goggle slippage [23]. DHI and vHIT were taken immediately before and after VR. The changes in DHI and VOR gain before and after rehabilitation were determined as $\triangle \mathrm{DHI}$ and $\triangle \mathrm{VOR}$, respectively. Collected data were analyzed in SPSS v.17 software using descriptive statistics (frequency, mean and standard deviation) and statistical tests including Kolmogorov-Smirnov test (for testing the normality of data distribution), 
Table 1. Vestibulocular reflex gain of semicircular canals and dizziness handicap inventory scores before and after vestibular rehabilitation

\begin{tabular}{|c|c|c|c|c|c|c|c|}
\hline & \multicolumn{3}{|l|}{ Pre-VR } & \multicolumn{3}{|l|}{ Post-VR } & \multirow[b]{2}{*}{$\mathbf{p}$} \\
\hline & Mean (SD) & Min & Max & Mean (SD) & Min & Max & \\
\hline VOR-ipsi horizontal SCC & $0.45(0.13)$ & 0.21 & 0.66 & $0.74(0.10)$ & 0.45 & 1.00 & $<0.001$ \\
\hline VOR-ipsi posterior SCC & $0.75(0.14)$ & 0.42 & 1.00 & $0.83(0.11)$ & 0.55 & 1.04 & $<0.001$ \\
\hline VOR-ipsi superior SCC & $0.75(0.15)$ & 0.42 & 1.04 & $0.82(0.10)$ & 0.57 & 1.00 & $<0.001$ \\
\hline VOR-contra horizontal SCC & $0.84(0.08)$ & 0.69 & 1.02 & $0.87(0.08)$ & 0.71 & 1.04 & $<0.001$ \\
\hline VOR-contra posterior SCC & $0.88(0.08)$ & 0.75 & 1.02 & $0.90(0.08)$ & 0.77 & 1.05 & $<0.001$ \\
\hline VOR-contra superior SCC & $0.89(0.08)$ & 0.75 & 1.01 & $0.91(0.08)$ & 0.77 & 1.09 & $<0.001$ \\
\hline DHI & $69.24(9.81)$ & 52 & 86 & $42.76(8.18)$ & 28 & 64 & $<0.001$ \\
\hline
\end{tabular}

VR; vestibular rehabilitation, VOR; vestibule-ocular reflex gain, SCC; semicircular canal, DHI; dizziness handicap inventory

Pearson correlation test, and paired sample t-test. $\mathrm{p}<0.05$ was set as the significance level.

\section{Results}

All patients completed the study. A significant increase in VOR gain from ipsilesional and contralesional SCCs and a significant decrease in DHI score after VR were reported $(\mathrm{p}<0.001)$ (Table 1). The $\Delta \mathrm{DHI}$ score had a significant strong negative correlation with $\triangle$ VOR from ipsilesional horizontal SCC $($ rho $=0.868)$ and a significant moderate negative correlation with $\triangle$ VOR from ipsilesional posterior $($ rho $=0.666)$ and superior SCCs (rho = 0.618, p < 0.001) (Fig. 1). However, no statistically significant correlations were found between the $\Delta \mathrm{DHI}$ and $\Delta \mathrm{VOR}$ from the contrlesional SCCs (Fig. 2)

\section{Discussion}

The present study was conducted to investigate the role of vHIT in VR follow-up and its correlation with DHI scores. We found a) a significant increase in VOR gain after VR in all three ipsilesional and contralesional SCCs, b) a significant correlation between $\triangle \mathrm{VOR}$ and $\triangle \mathrm{DHI}$ in all three ipsilesional SCCs, and c) no correlation between $\triangle \mathrm{VOR}$ and $\triangle \mathrm{DHI}$ in contralesional SCCs. The first finding of this study is in agreement with previous studies in this area $[7,11,15]$.
It is noteworthy that previous studies had only examined individuals with ipsilesional horizontal SCC, while our study examined all three SCCs and showed improvement in VOR gain of UPVH patients from both ipsilesional and contralesional SCCs. Since bilateral symmetry of VOR gain plays an important role in controlling the posture and visual stability during head movements, assessment of all six SCCs is recommended. vHIT for all three SCCs is also essential for monitoring the VR process in the subacute phase, considering that the focus of most VR exercises in UPVH patients is on their visual stability, since their visual acuity decreases during head movements.

The main finding of this study was a significant negative correlation between the increase in VOR gain and the decrease in DHI scores in all three ipsilesional SCCs after VR. This means that with functional improvement in the SCCs (VOR gain), dizziness-related disability (DHI score) decreases. The correlation between absolute DHI scores and balance test scores has been investigated only by a few studies which reported a weak correlation between DHI scores and the results of vestibular tests such as rotary chair test, electronystagmography, posturography, caloric, vestibular evoked myogenic potential, and vHIT $[10,16,24,25]$. Jacobson and McCaslin examined 

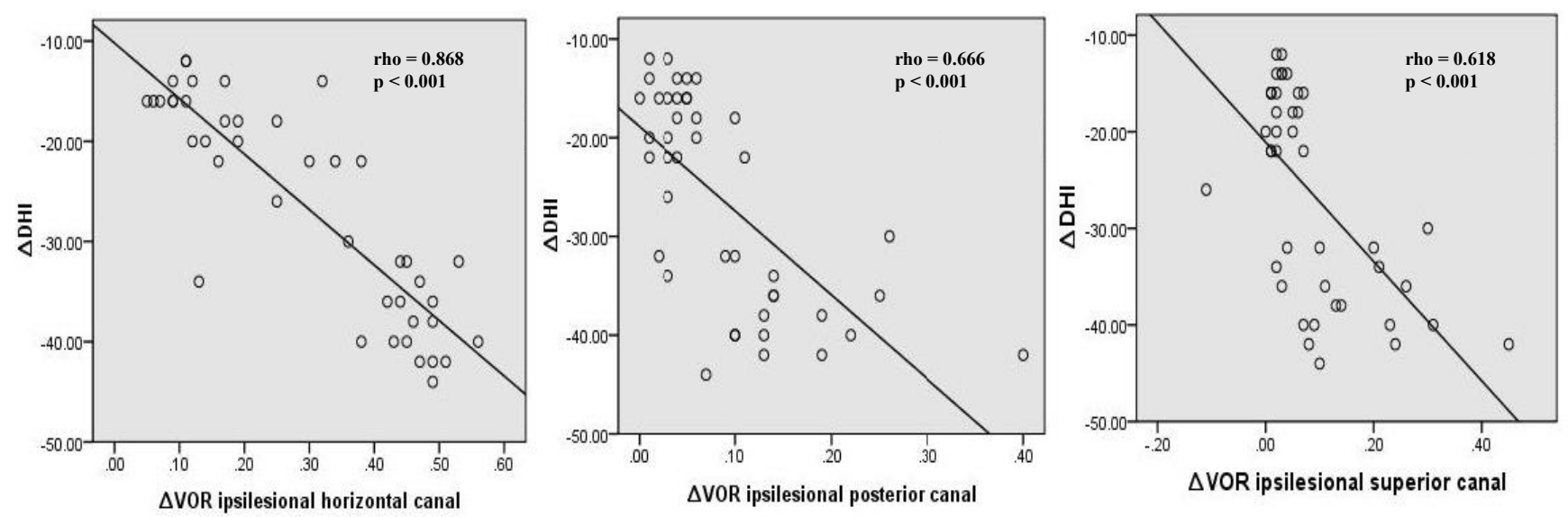

Fig. 1. Scatter plots showing correlation between improvement of the dizziness handicap, inventory scores and of the vestibule-ocular reflex gains in ipsilesional canals before and after vestibular rehabilitation. $\triangle \mathrm{DHI}$; dizziness handicap inventory improvement post vestibular rehabilitation, $\Delta$ VOR; vestibule-ocular reflex gain improvement post vestibular rehabilitation.

unilateral compensation for vestibular weakness; however, their study did not control the effect of age and included individuals aged $>60$ years [25]. McCalsin et al. included all patients with vertigo symptoms and no specific group was used [16]. Yip and Strupp studied the patients with chronic vestibular lesions for at least three months [10]. Abnormally high DHI scores were also observed in older patients with neck problems and neuropathies due to diabetes [25]. In contrast, lower DHI scores were reported in a study for those with chronic phase of vestibular lesions due to their compensatory mechanisms [16]. Therefore, the lack of correlation between the DHI scores and vestibular test results is likely due to the lack of control over the aforementioned factors, which may strongly affect the DHI scores. We studied patients with subacute UPVH who were between 20 and 60 years of age and had no history of neck problems and diabetes; therefore, by controlling the factors affecting the DHI score, a stronger correlation was observed between $\triangle$ VOR gain and $\triangle \mathrm{DHI}$ score. Our results were consistent with other studies that reported a significant correlation between absolute DHI scores and balance tests [26,27]. To our knowledge, this is the first study that evaluates the correlation between DHI score and VOR gain and provides a better knowledge of the
VR follow-up results. The lack of correlation between DHI score and VOR gain from all three contralesional SCCs suggested that vHIT results for the contralesional side could not predict the degree of disability from these lesions. This result was expected because the opposite side is not affected in patients with unilateral hypofunction. The strong correlation between DHI score and VOR gain in the three ipsilesional SCCs, especially the horizontal ones, indicates that the improvement in vHIT results not only provides useful information about improved subacute dysfunction of the vestibular organ but also improves the ability to perform daily living activities and the disability caused by vestibular damage. This test examines eye movements at high head velocities, which is equivalent to the speed of head movements in daily living activities. From this perspective, the improvement in vHIT scores in all three SCCs after VR may be a good predictor of the degree of improvement in dizzinessrelated disability.

This study had some limitations. Due to the proven effectiveness of VR in UPVH patients, we did not use a control group. The aim of this study was not only to confirm the effectiveness of VR, but also to describe the changes in VOR gain after VR. We did not carry out the otolith function testing and did not examine the 

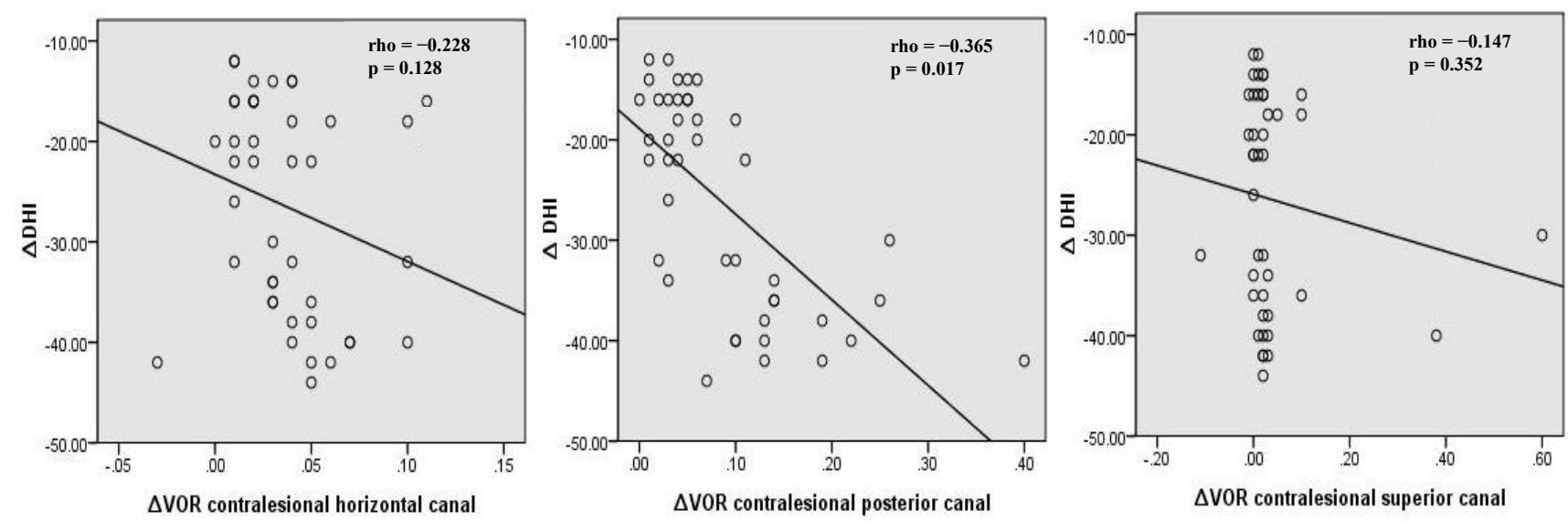

Fig. 2. Scatter plots showing the correlation between improvements of the dizziness handicap inventory scores and the vestibule-ocular reflex gains in contralesional canals before and after vestibular rehabilitation. $\triangle \mathrm{DHI}$; dizziness handicap inventory improvement post vestibular rehabilitation, $\triangle$ VOR; vestibule-ocular reflex gain improvement post vestibular rehabilitation.

association of injuries in otolith organs with our results. The final limitation was the lack of information on the corrective saccades in the vHIT test, which was not investigated due to data overload.

\section{Conclusion}

The video head impulse test(vHIT) is a useful tool to evaluate the effectiveness of vestibular rehabilitation (VR). Vestibulo-ocular reflex gain is correlated with the dizziness handicap inventory score. Therefore, the improvement in vHIT results in all three ipsilesional semicircular canals after VR may be a good predictor of the degree of improvement in dizziness-related disability.

\section{Acknowledgments}

This study is the result of the research project No. 99-1-6-17982 at Iran University of Medical Sciences. The authors would like to thank participants in this research.

\section{Conflict of interest}

The authors declare no conflict of interest.

\section{References}
1. Agrawal Y, Ward BK, Minor LB. Vestibular dysfunction: prevalence, impact and need for targeted treatment. J Vestib Res. 2013;23(3):113-7. doi: 10.3233/VES-130498 Bronstein AM, Golding JF, Gresty MA, Mandalà M, Nuti

$\mathrm{D}$, Shetye A, et al. The social impact of dizziness in London and Siena. J Neurol. 2010;257(2):183-90. doi: 10.1007/s00415-009-5287-z

3. Herdman SJ, Blatt P, Schubert MC, Tusa RJ. Falls in patients with vestibular deficits. Am J Otol. 2000;21(6): 847-51.

4. Herdman SJ, Schubert MC, Das VE, Tusa RJ. Recovery of dynamic visual acuity in unilateral vestibular hypofunction. Arch Otolaryngol Head Neck Surg. 2003;129 (8):819-24. doi: 10.1001/archotol.129.8.819

5. Strupp M, Magnusson M. Acute unilateral vestibulopathy. Neurol Clin. 2015;33(3):669-85, x. doi: 10.1016/j.ncl.2015.04.012

6. Schubert MC, Migliaccio AA, Clendaniel RA, Allak A, Carey JP. Mechanism of dynamic visual acuity recovery with vestibular rehabilitation. Arch Phys Med Rehabil. 2008;89(3):500-7. doi: 10.1016/j.apmr.2007.11.010

7. Sjögren J, Fransson PA, Karlberg M, Magnusson M, Tjernström F. Functional head impulse testing might be useful for assessing vestibular compensation after unilateral vestibular loss. Front Neurol. 2018;9:979. doi: 10.3389/fneur.2018.00979

8. Herdman SJ. Vestibular rehabilitation. Current opinion in neurology. 2013;26(1):96-101. doi: 10.1097/WCO.0b013e32835c5ec4

9. Rosiak O, Szczepanik M, Woszczak M, LucasGrzelczyk W, Józefowicz-Korczyńska M. [Effectiveness of vestibular rehabilitation in patients with vestibular dysfunction]. Med Pr. 2019;70(5):545-53. Polish. doi: 10.13075/mp.5893.00853

10. Yip CW, Strupp M. The dizziness handicap inventory does not correlate with vestibular function tests: a prospective study. J Neurol. 2018;265(5):1210-18. doi: 10.1007/s00415-018-8834-7

11. Viziano A, Micarelli A, Augimeri I, Micarelli D, Alessandrini M. Long-term effects of vestibular rehabilitation and head-mounted gaming task procedure in unilateral vestibular hypofunction: a 12-month follow-up of a randomized controlled trial. Clin Rehabil. 2019;33(1):24-33. doi: $10.1177 / 0269215518788598$ 
12. Topuz O, Topuz B, Ardiç FN, Sarhus M, Ögmen G, Ardiç F. Eficacy of vestibular rehabilitation on chronic unilateral vestibular dysfunction. Clin Rehabil. 2004;18(1): 76-83. doi: 10.1191/0269215504cr704oa

13. Son EJ, Lee DH, Oh JH, Seo JH, Jeon EJ. Correlation between the dizziness handicap inventory and balance performance during the acute phase of unilateral vestibulopathy. Am J Otolaryngol. 2015;36(6):823-7. doi: 10.1016/j.amjoto.2015.07.011

14. Formeister EJ, Krauter R, Kirk L, Zhu TR, Rizk HG, Sharon JD. Understanding the dizziness handicap inventory (DHI): a cross sectional analysis of symptom factors that contribute to DHI variance. Otol Neurotol. 2020; 41(1):86-93. doi: 10.1097/MAO.0000000000002438

15. Navari E, Cerchiai N, Casani AP. Assessment of vestibulo-ocular reflex gain and catch-up saccades during vestibular rehabilitation. Otol Neurotol. 2018;39(10): e1111-7. doi: 10.1097/MAO.0000000000002032

16. McCaslin DL, Jacobson GP, Bennett ML, Gruenwald JM, Green AP. Predictive properties of the video head impulse test: measures of caloric symmetry and self-report dizziness handicap. Ear Hear. 2014;35(5):e185-91. doi: 10.1097/AUD.0000000000000047

17. Robertson DD, Ireland DJ. Dizziness handicap inventory correlates of computerized dynamic posturography. J Otolaryngol. 1995;24(2):118-24.

18. Halmagyi GM, Curthoys IS. The video head impulse test in clinical practice. Neurol Sci Neurophysiol. 2018; 35(1):1-5.

19. Herdman SJ, Schubert MC, Das VE, Tusa RJ. Recovery of dynamic visual acuity in unilateral vestibular hypofunction. Arch Otolaryngol Head Neck Surg. 2003;129 (8):819-24. doi: 10.1001/archotol.129.8.819

20. Jafarzadeh S, Bahrami E, Pourbakht A, Jalaie S, Daneshi A. Validity and reliability of the Persian version of the dizziness handicap inventory. J Res Med Sci. 2014;19(8): 769-75.

21. Jacobson GP, Newman CW. The development of the dizziness handicap inventory. Arch Otolaryngol Head Neck Surg. 1990;116(4):424-7. doi: 10.1001/archotol.1990.01870040046011

22. Murnane O, Mabrey H, Pearson A, Byrd S, Akin F. Normative data and test-retest reliability of the SYNAPSYS video head impulse test. J Am Acad Audiol. 2014;25(3):244-52. doi: 10.3766/jaaa.25.3.3

23. Curthoys IS, Manzari L. Clinical application of the head impulse test of semicircular canal function. Hearing Balance Commun. 2017;15(3):113-26. doi: 10.1080/21695717.2017.1353774

24. Redondo-Martínez J, Bécares-Martínez C, Orts-Alborch M, García-Callejo FJ, Pérez-Carbonell T, Marco-Algarra J. Relationship between video head impulse test (vHIT) and caloric test in patients with vestibular neuritis. Acta Otorrinolaringol Esp. 2016;67(3):156-61. doi: 10.1016/j.otorri.2015.07.005

25. Jacobson GP, McCaslin DL. Agreement between functional and electrophysiologic measures in patients with unilateral peripheral vestibular system impairment. J Am Acad Audiol. 2003;14(5):231-8.

26. Batuecas-Caletrio A, Santacruz-Ruiz S, MuñozHerrera A, Perez-Fernandez N. The vestibulo-ocular reflex and subjective balance after vestibular schwannoma surgery. Laryngoscope. 2014;124(6):1431-5. doi: 10.1002/lary.24447

27. Eravcı FC, Yılmaz M, Uğur MB, Tutar H, Karamert $\mathrm{R}$, Şansal E, et al. The Relationship of vestibuloocular reflex with self-reported dizziness handicap in patients with vestibular deafferentation. Ear Nose Throat J. 2019;145561319880028. doi: $10.1177 / 0145561319880028$ 